\title{
Maxillary and mandibular anterior crown width/height ratio and its relation to various arch perimeters, arch length, and arch width groups
}

\author{
Fazal Shahid ${ }^{1}$, Mohammad Khursheed Alam ${ }^{1}$, Mohd Fadhli Khamis ${ }^{2}$
}

Correspondence: Dr. Mohammad Khursheed Alam

Email:dralam@gmail.com

\author{
1Orthodontic Unit, School of Dental Science, Universiti \\ Sains Malaysia, Kelantan, Kubang Kerian, 16150, \\ Malaysia, \\ 2Forensic Dentistry Unit, School of Dental Science, \\ Universiti Sains Malaysia, Kelantan, Kubang Kerian, \\ 16150, Malaysia
}

\section{ABSTRACT}

Objective: To investigate the maxillary and mandibular anterior crown width/height ratio and its relation to various arch perimeters, arch length, and arch width (intercanine, interpremolar, and intermolar) groups. Materials and Methods: The calculated sample size was 128 subjects. The crown width/height, arch length, arch perimeter, and arch width of the maxilla and mandible were obtained via digital calliper (Mitutoyo, Japan). A total of 4325 variables were measured. The sex differences in the crown width and height were evaluated. Analysis of variance was applied to evaluate the differences between arch length, arch perimeter, and arch width groups. Results: Males had significantly larger mean values for crown width and height than females $(P \leq 0.05)$ for maxillary and mandibular arches, both. There were no significant differences observed for the crown width/height ratio in various arch length, arch perimeter, and arch width (intercanine, interpremolar, and intermolar) groups $(P \leq 0.05)$ in maxilla and mandible, both. Conclusions: Our results indicate sexual disparities in the crown width and height. Crown width and height has no significant relation to various arch length, arch perimeter, and arch width groups of maxilla and mandible. Thus, it may be helpful for orthodontic and prosthodontic case investigations and comprehensive management.

Key words: Arch length, arch perimeter, arch width, crown height, crown width/height ratio, crown width

\section{INTRODUCTION}

Variations in tooth size, tooth morphology, and tooth size ratio have been connected with diverse ethnic foundations and occlusion statuses. ${ }^{[1-9]}$ Sexual dimorphism in crown dimension has relation to humanoid sex genes and hormones and is influenced by their imbalance. ${ }^{[10]}$ Hereditary and ecological factors have strong effects on human teeth. ${ }^{[11]}$ Correspondingly, the teeth width/height ratio has population and ethnic variations in relation to shape. ${ }^{[12]}$ These ethnic variations must be considered in treatment planning especially to address esthetic

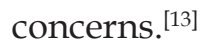

Harmonious anterior teeth with proper size and shape were one of the most influential factors contributing to a pleasant smile in orthodontic, operative, and prosthodontic management. Lombardi ${ }^{[14]}$ was the first treatment providers to accentuate the status of dental proportions with different facial types. They mentioned that there was a recurring ratio noted between all teeth in relation with face from the central incisor to the first premolar. Levin ${ }^{[15]}$ and Qualtrough and Burke ${ }^{[16]}$ indicated that the most harmonious teeth proportion is 1:1.618 between central incisor to lateral incisor. However, conflicting reports ${ }^{[17-19]}$ indicated that the

This is an open access article distributed under the terms of the Creative Commons Attribution-NonCommercial-ShareAlike 3.0 License, which allows others to remix, tweak, and build upon the work non-commercially, as long as the author is credited and the new creations are licensed under the identical terms.

For reprints contact: reprints@medknow.com

How to cite this article: Shahid F, Alam MK, Khamis MF. Maxillary and mandibular anterior crown width/height ratio and its relation to various arch perimeters, arch length, and arch width groups. Eur J Dent 2015;9:490-9.

DOI: $10.4103 / 1305-7456.172620$ 
majority of beautiful smiles did not have these dental proportions. Several anatomic measurements have been proposed to aid in determining the correct size and shape of the anterior teeth in relation to the various facial land marks and types on various populations. ${ }^{[20-24]}$

The authors have identified that no relative analysis of the maxillary and mandibular anterior tooth width/height ratios in relation to various arch perimeters, arch length, and arch width (intercanine, interpremolar, and intermolar) groups for the Pakistani population has been previously done.

The purpose of this study was:

- To evaluate the anatomic crown width/height ratios of maxillary and mandibular anterior tooth for sexual dimorphism

- To evaluate the width/height ratios of maxillary and mandibular anterior tooth in relation to various arch perimeter groups

- To evaluate the width/height ratios of maxillary and mandibular anterior tooth in relation to various arch length groups

- To evaluate the width/height ratios of maxillary and mandibular anterior tooth in relation to various arch width groups:

- Intercanine width

- Interpremolar width

- Intermolar width.

\section{MATERIALS AND METHODS}

\section{Subjects}

The study was a comparative retrospective design. The oral and dental investigations were carried out with careful selection of subjects from the Pakistani population. Ethical approval was granted by the Ethics Committee of the Universiti Sains Malaysia (USM/ JEPeM/140376) and informed consent was obtained from subjects. This investigation was designed and conducted according to the guidelines of Strengthening the Reporting of Observational studies in Epidemiology (STROBE), and we applied the STROBE specification in this manuscript. ${ }^{[25]}$

\section{Sample size calculation}

The sample size was calculated at a power of $80 \%$, utilizing estimated standard deviations (SDs) of $0.60 \mathrm{~mm},{ }^{[26]}$ a biologically meaningful mean difference of $0.3 \mathrm{~mm}$, and equal sample sizes. ${ }^{[27]}$ The calculated sample size was 128 subjects (64 males and 64 females with a mean age 19.4 \pm 1.9 SD). The following inclusion and exclusion criteria were used.

\section{Inclusion criteria}

- All patients were of Pakistani origin determined via interviews, with mutual paternities and ancestors without any multiethnic nuptials

- Subjects were aged 18-24 years

- Maxillary and mandibular well-aligned arches, with normal patterns of growth and development

- None of the participants had undergone orthodontic treatment, with all sound erupted permanent teeth (except third molars)

- Ideal occlusion with Class I molar and canine relationship with incisors according to the British Standards Institute ${ }^{[28]}$

- No crowding, cross bite and spacing

- Straight profile (identified by examining the profile view)

- No craniofacial anomalies

- No gingival or periodontal conditions or therapy that would undermine a healthy tissue-to-tooth relationship.

\section{Exclusion criteria}

- Interproximal caries or restorations

- Missing or supernumerary teeth

- Abnormal size or morphology of teeth

- Tooth wear that affected the tooth size measurements

- Damage to casts.

Cross-examination of subjects was done to diminish sample bias and error; with an experienced orthodontist and dentist contributing throughout the screening sittings. Dental impressions of the upper and lower arches of each subject were obtained with alginate impression material (Zhermack Orthoprint Alginate ISO 1563-ADA 18 Italy) and poured with dental stone (Type III hard plaster quick stone China) according to the manufacturer's instructions. A total of 4325 variables were measured.

\section{Measurement of crown width, crown height and arch dimensions}

Dental models of each subject for maxillary and mandibular arches were carefully selected according to inclusion and exclusion criteria. Crown width, crown height, arch length, arch perimeter, and arch width of the maxilla and mandible were obtained via digital caliper (Mitutoyo, Japan) ${ }^{[29]}$ as follows.

\section{Crown width measurement}

The mesiodistal crown diameter of the tooth was measured from anatomical contact of one tooth to another from frontal side perpendicular to the long axis of the teeth. ${ }^{[12]}$ 


\section{Crown height measurement}

Crown height [Figure 1] was recorded as the greatest distance on buccal/labial surface from the occlusal/ incisal line to cervical line parallel to the occlusal plane. ${ }^{[12,30]}$

\section{Arch dimensions measurements}

\section{Arch perimeter}

Arch perimeter ${ }^{[2,31]}$ was measured as a segmental sum of linear lines on the right and left side of the arches [Figure 2c].

\section{Arch length}

Arch length was obtained using triangular shaped lines between the mesiobuccal cusp tips of first permanent molars and the central point between the incisors of each respective arch [Figure 2b].

Arch width of maxillary and mandibular variables

- Maxillary and mandibular intercanine widths were obtained between the cusp tips

- Maxillary and mandibular interpremolar widths were obtained between the cusp tips of maxillary first premolars

- Maxillary and mandibular intermolar widths were obtained between the mesiobuccal cusp tips of the maxillary and mandibular first molars respectively [Figure 2a].

Arch length, arch perimeter, and arch width grouping The subjects were further grouped as follows:

- Arch length groups (small, average, and large): The numbers of subjects for small, average, and large arch length groups in the maxilla and mandible were 46,44 , and 38 , respectively

- Arch perimeter groups (small, average, and large): The number of subject for small, average, and large arch perimeter groups in the maxilla and mandible

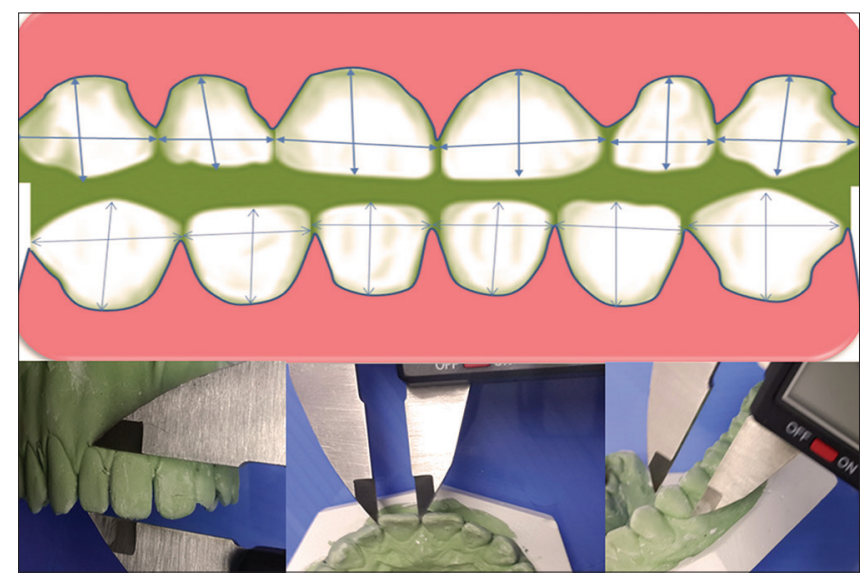

Figure 1: Crown width and height measurement via digital caliper were 44,45 , and 39 , respectively

- Arch widths group (small, average, and large): The number of subject for small, average, and large arch width groups in the maxilla and mandible were 44,45 , and 39, respectively (intercanine, interpremolar, and intermolar width).

These groupings were determined based on data values of the mean $\pm 2 \mathrm{SD},>2 \mathrm{SD}$, and $<2 \mathrm{SD}$ grouped in the average group, large group, and small group, respectively. ${ }^{[31]}$

\section{Error study}

Twenty percentage of dental casts were randomly selected for intraobserver errors. The time interval between the first and second readings was approximately 2 weeks. The method error (ME) was analyzed by the Dalhberg's formula:

$\mathrm{ME}=\left(\Sigma\left[\mathrm{x}_{1}-\mathrm{x}_{2}\right]^{2} / 2[2 \mathrm{n}]\right)^{1 / 2}$

Where $x_{1}$ is the first measurement, $x_{2}$ the second measurement and $n$ the number of repeated measurements. ${ }^{[32]}$

\section{Statistical analyses}

The data were verified and analyzed statistically using IBM SPSS Statistics version 22.0 (Armonk, NY: IBM Corp., USA) with the confidence level set at $5 \%(P<0.05)$ to test for significance. Independent $t$-tests were applied to compare mean values between males and females crown width, crown height, and crown width/ height ratio in relation to all variables. Analysis of variance was applied to evaluate crown width/height ratio in relation to the arch length, arch perimeter, and arch widths groups. The post-hoc

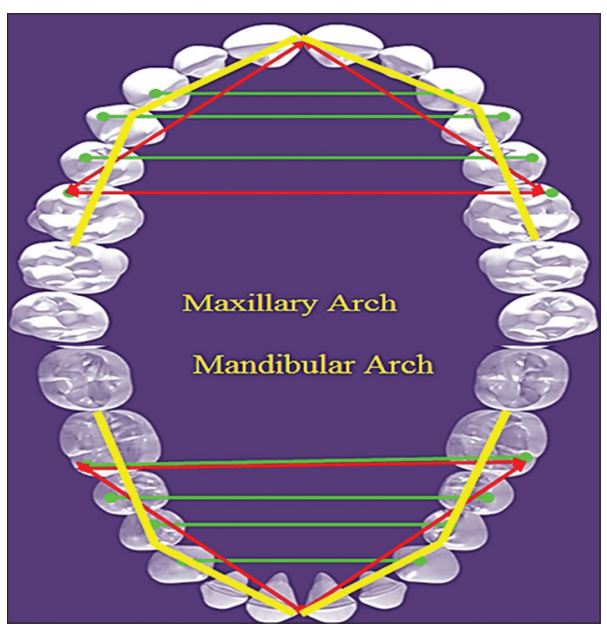

Figure 2: Arch dimension measurements (a) arch width (Green color) (b) arch length (yellow color) (c) arch perimeter (Red color) 


\begin{tabular}{|c|c|c|c|c|c|c|}
\hline \multirow[t]{2}{*}{ Variables* } & \multirow[t]{2}{*}{ Sex } & \multirow[t]{2}{*}{ Mean } & \multirow[t]{2}{*}{ SD } & \multicolumn{2}{|c|}{$95 \% \mathrm{Cl}$} & \multirow[t]{2}{*}{$P$} \\
\hline & & & & Lower & Upper & \\
\hline \multirow[t]{2}{*}{ WHR11 } & Male & 0.93 & 0.09 & -0.04 & 0.02 & 0.544 \\
\hline & Female & 0.94 & 0.09 & -0.04 & 0.02 & \\
\hline \multirow[t]{2}{*}{ WHR12 } & Male & 0.89 & 0.11 & -0.04 & 0.03 & 0.907 \\
\hline & Female & 0.89 & 0.09 & -0.04 & 0.03 & \\
\hline \multirow[t]{2}{*}{ WHR13 } & Male & 0.90 & 0.10 & -0.09 & -0.01 & 0.011 \\
\hline & Female & 0.95 & 0.11 & -0.09 & -0.01 & \\
\hline \multirow[t]{2}{*}{ WHR21 } & Male & 0.93 & 0.09 & -0.03 & 0.03 & 0.982 \\
\hline & Female & 0.93 & 0.08 & -0.03 & 0.03 & \\
\hline \multirow[t]{2}{*}{ WHR22 } & Male & 0.88 & 0.10 & -0.03 & 0.03 & 0.977 \\
\hline & Female & 0.88 & 0.09 & -0.03 & 0.03 & \\
\hline \multirow[t]{2}{*}{ WHR23 } & Male & 0.90 & 0.10 & -0.07 & 0.00 & 0.085 \\
\hline & Female & 0.93 & 0.11 & -0.07 & 0.00 & \\
\hline \multirow[t]{2}{*}{ WHR31 } & Male & 0.74 & 0.08 & -0.06 & 0.00 & 0.041 \\
\hline & Female & 0.77 & 0.09 & -0.06 & 0.00 & \\
\hline \multirow[t]{2}{*}{ WHR32 } & Male & 0.87 & 0.55 & -0.10 & 0.18 & 0.551 \\
\hline & Female & 0.83 & 0.10 & -0.10 & 0.18 & \\
\hline \multirow[t]{2}{*}{ WHR33 } & Male & 0.81 & 0.10 & -0.04 & 0.03 & 0.685 \\
\hline & Female & 0.81 & 0.10 & -0.04 & 0.03 & \\
\hline \multirow[t]{2}{*}{ WHR41 } & Male & 0.74 & 0.08 & -0.05 & 0.01 & 0.115 \\
\hline & Female & 0.77 & 0.09 & -0.05 & 0.01 & \\
\hline \multirow[t]{2}{*}{ WHR42 } & Male & 0.86 & 0.50 & -0.09 & 0.17 & 0.522 \\
\hline & Female & 0.82 & 0.08 & -0.09 & 0.17 & \\
\hline \multirow[t]{2}{*}{ WHR43 } & Male & 0.80 & 0.10 & -0.04 & 0.02 & 0.580 \\
\hline & & 0.81 & 0.09 & -0.04 & 0.02 & \\
\hline
\end{tabular}

${ }^{*}$ FDI notation, $(P \leq 0.001),(P \leq 0.01)$ and $(P \leq 0.05)$. WHR: Width/height ratio, $\mathrm{Cl}$ : Confidence interval, SD: Standard deviation, FDI: Federation Dentaire Internationale tests of Bonferroni correction were performed for differences among the groups.

\section{RESULTS}

\section{Method error}

Dahlberg's formula was used to determine the ME, which did not exceed 0.006 and $0.05 \mathrm{~mm}$ for the linear variables of teeth crown width and crown height, respectively. The combined errors for all of the variables were small and considered to be within acceptable limits. ${ }^{[32]}$

\section{Sexual disparities in crown width/height ratios} Table 1 shows no significant difference for the crown width/height ratios, except the maxillary right canine (WHR13) and mandibular left central incisor(WHR31).

Disparities in relation to arch length, arch perimeter, and arch widths groups of the maxilla

Tables 2-6 show the maxillary arch perimeter, arch length, and arch width (intercanine, interpremolar, and intermolar) groups in relation to crown width/height ratios respectively. There were no significant differences observed in relation to all groups $(P \leq 0.05)$. Except few variables for the intercanine and inter first molar arch width for the

\begin{tabular}{|c|c|c|c|c|c|c|c|c|}
\hline \multirow[t]{2}{*}{ Variables } & \multirow[t]{2}{*}{ UAPG } & \multirow[t]{2}{*}{ Mean } & \multirow[t]{2}{*}{ SD } & \multicolumn{2}{|c|}{$95 \% \mathrm{Cl}$} & \multicolumn{3}{|c|}{$P$} \\
\hline & & & & Lower & Upper & 1 versus 2 & 1 versus 3 & 2 versus 3 \\
\hline \multirow[t]{3}{*}{ WHR11 } & 1.00 & 0.93 & 0.08 & 0.91 & 0.96 & 0.61 & 1.00 & 0.55 \\
\hline & 2.00 & 0.92 & 0.09 & 0.90 & 0.95 & & & \\
\hline & 3.00 & 0.95 & 0.09 & 0.92 & 0.98 & & & \\
\hline \multirow[t]{3}{*}{ WHR12 } & 1.00 & 0.89 & 0.08 & 0.86 & 0.91 & 0.24 & 1.00 & 1.00 \\
\hline & 2.00 & 0.88 & 0.11 & 0.84 & 0.91 & & & \\
\hline & 3.00 & 0.90 & 0.11 & 0.86 & 0.93 & & & \\
\hline \multirow[t]{3}{*}{ WHR13 } & 1.00 & 0.93 & 0.10 & 0.90 & 0.96 & 1.00 & 1.00 & 1.00 \\
\hline & 2.00 & 0.93 & 0.11 & 0.90 & 0.96 & & & \\
\hline & 3.00 & 0.92 & 0.13 & 0.88 & 0.97 & & & \\
\hline \multirow[t]{3}{*}{ WHR21 } & 1.00 & 0.93 & 0.08 & 0.91 & 0.96 & 0.39 & 1.00 & 0.85 \\
\hline & 2.00 & 0.92 & 0.09 & 0.90 & 0.95 & & & \\
\hline & 3.00 & 0.94 & 0.09 & 0.91 & 0.97 & & & \\
\hline \multirow[t]{3}{*}{ WHR22 } & 1.00 & 0.87 & 0.08 & 0.85 & 0.90 & 0.07 & 1.00 & 0.34 \\
\hline & 2.00 & 0.87 & 0.10 & 0.84 & 0.90 & & & \\
\hline & 3.00 & 0.89 & 0.11 & 0.86 & 0.93 & & & \\
\hline \multirow[t]{3}{*}{ WHR23 } & 1.00 & 0.91 & 0.10 & 0.88 & 0.94 & 1.00 & 1.00 & 1.00 \\
\hline & 2.00 & 0.93 & 0.09 & 0.90 & 0.96 & & & \\
\hline & 3.00 & 0.91 & 0.11 & 0.87 & 0.95 & & & \\
\hline
\end{tabular}


Shahid, et al.: Crown width/height ratios and its relation various arch groups

\begin{tabular}{|c|c|c|c|c|c|c|c|c|}
\hline \multirow[t]{2}{*}{ Variables } & \multirow[t]{2}{*}{ UALG } & \multirow[t]{2}{*}{ Mean } & \multirow[t]{2}{*}{ SD } & \multicolumn{2}{|c|}{$95 \% \mathrm{Cl}$} & \multicolumn{3}{|c|}{$P$} \\
\hline & & & & Lower & Upper & 1 versus 2 & 1 versus 3 & 2 versus 3 \\
\hline \multirow[t]{3}{*}{ WHR11 } & 1.00 & 0.93 & 0.08 & 0.91 & 0.96 & 1.000 & 1.000 & 0.684 \\
\hline & 2.00 & 0.92 & 0.09 & 0.90 & 0.95 & & & \\
\hline & 3.00 & 0.95 & 0.09 & 0.92 & 0.98 & & & \\
\hline \multirow[t]{3}{*}{ WHR12 } & 1.00 & 0.89 & 0.08 & 0.86 & 0.91 & 1.000 & 1.000 & 1.000 \\
\hline & 2.00 & 0.88 & 0.11 & 0.84 & 0.91 & & & \\
\hline & 3.00 & 0.90 & 0.11 & 0.86 & 0.93 & & & \\
\hline \multirow[t]{3}{*}{ WHR13 } & 1.00 & 0.93 & 0.10 & 0.90 & 0.96 & 1.000 & 1.000 & 1.000 \\
\hline & 2.00 & 0.93 & 0.11 & 0.90 & 0.96 & & & \\
\hline & 3.00 & 0.92 & 0.13 & 0.88 & 0.97 & & & \\
\hline \multirow[t]{3}{*}{ WHR21 } & 1.00 & 0.93 & 0.08 & 0.91 & 0.96 & 1.000 & 1.000 & 1.000 \\
\hline & 2.00 & 0.92 & 0.09 & 0.90 & 0.95 & & & \\
\hline & 3.00 & 0.94 & 0.09 & 0.91 & 0.97 & & & \\
\hline \multirow[t]{3}{*}{ WHR22 } & 1.00 & 0.87 & 0.08 & 0.85 & 0.90 & 1.000 & 0.914 & 0.839 \\
\hline & 2.00 & 0.87 & 0.10 & 0.84 & 0.90 & & & \\
\hline & 3.00 & 0.89 & 0.11 & 0.86 & 0.93 & & & \\
\hline \multirow[t]{3}{*}{ WHR23 } & 1.00 & 0.91 & 0.10 & 0.88 & 0.94 & 1.000 & 1.000 & 1.000 \\
\hline & 2.00 & 0.93 & 0.09 & 0.90 & 0.96 & & & \\
\hline & 3.00 & 0.91 & 0.11 & 0.87 & 0.95 & & & \\
\hline
\end{tabular}

${ }^{*} \mathrm{FDI}$, notation, $P \leq 0.001, P \leq 0.01$ and $P \leq 0.05$. UALG: Upper arch length groups, WHR: Width/height ratio, 1: Small, 2: Average, 3: Large, Cl: Confidence interval, SD: Standard deviation, FDI: Federation Dentaire Internationale

\begin{tabular}{|c|c|c|c|c|c|c|c|c|}
\hline \multirow[t]{2}{*}{ Variables } & \multirow[t]{2}{*}{ UAICWG } & \multirow[t]{2}{*}{ Mean } & \multirow[t]{2}{*}{ SD } & \multicolumn{2}{|c|}{$95 \% \mathrm{Cl}$} & \multicolumn{3}{|c|}{$P$} \\
\hline & & & & Lower & Upper & 1 versus 2 & 1 versus 3 & 2 versus 3 \\
\hline \multirow[t]{3}{*}{ WHR11 } & 1.00 & 0.93 & 0.07 & 0.91 & 0.95 & 0.212 & 0.070 & 0.000 \\
\hline & 2.00 & 0.90 & 0.09 & 0.87 & 0.93 & & & \\
\hline & 3.00 & 0.97 & 0.09 & 0.94 & 1.00 & & & \\
\hline \multirow[t]{3}{*}{ WHR12 } & 1.00 & 0.87 & 0.09 & 0.84 & 0.90 & 1.000 & 0.014 & 0.002 \\
\hline & 2.00 & 0.86 & 0.08 & 0.83 & 0.88 & & & \\
\hline & 3.00 & 0.93 & 0.11 & 0.90 & 0.97 & & & \\
\hline \multirow[t]{3}{*}{ WHR13 } & 1.00 & 0.92 & 0.09 & 0.89 & 0.95 & 1.000 & 0.278 & 0.068 \\
\hline & 2.00 & 0.90 & 0.11 & 0.87 & 0.94 & & & \\
\hline & 3.00 & 0.96 & 0.12 & 0.92 & 1.00 & & & \\
\hline \multirow[t]{3}{*}{ WHR21 } & 1.00 & 0.93 & 0.07 & 0.91 & 0.95 & 0.158 & 0.039 & 0.000 \\
\hline & 2.00 & 0.90 & 0.08 & 0.87 & 0.92 & & & \\
\hline & 3.00 & 0.97 & 0.09 & 0.94 & 1.00 & & & \\
\hline \multirow[t]{3}{*}{ WHR22 } & 1.00 & 0.87 & 0.09 & 0.85 & 0.90 & 0.574 & 0.068 & 0.002 \\
\hline & 2.00 & 0.85 & 0.08 & 0.82 & 0.87 & & & \\
\hline & 3.00 & 0.92 & 0.11 & 0.88 & 0.95 & & & \\
\hline \multirow[t]{3}{*}{ WHR23 } & 1.00 & 0.91 & 0.09 & 0.89 & 0.94 & 1.000 & 0.493 & 0.082 \\
\hline & 2.00 & 0.89 & 0.10 & 0.86 & 0.92 & & & \\
\hline & 3.00 & 0.94 & 0.12 & 0.91 & 0.98 & & & \\
\hline
\end{tabular}

${ }^{*}$ FDI, notation, $P \leq 0.001, P \leq 0.01$ and $P \leq 0.05$. UAICWG: Upper arch intercanine width groups, WHR: Width/height ratio, 1: Small, 2: Average, 3: Large, Cl: Confidence interval, SD: Standard deviation, FDI: Federation Dentaire Internationale

small versus large and average versus large group were observed $(P \leq 0.001),(P \leq 0.01)$, and $(P \leq 0.05)$.

Disparities in relation to arch length, arch perimeter, and arch widths groups of the mandible

Tables 7-11 show the mandibular arch perimeter, arch length, and arch width (intercanine, interpremolar, and intermolar) groups in relation to crown width/height ratios, respectively. There were no significant differences observed in all groups $(P \leq 0.05)$.

Correlation for width/height ratio and its relation to various arch dimensions

Table 12 shows the correlation coefficients determined between the measured maxillary and mandibular crown width/ height ratios values and the 
Shahid, et al.: Crown width/height ratios and its relation various arch groups

\begin{tabular}{|c|c|c|c|c|c|c|c|c|}
\hline \multirow[t]{2}{*}{ Variables } & \multirow[t]{2}{*}{ UAIPMWG } & \multirow[t]{2}{*}{ Mean } & \multirow[t]{2}{*}{ SD } & \multicolumn{2}{|c|}{$95 \% \mathrm{Cl}$} & \multicolumn{3}{|c|}{$P$} \\
\hline & & & & Lower & Upper & 1 versus 2 & 1 versus 3 & 2 versus 3 \\
\hline \multirow[t]{3}{*}{ WHR11 } & 1.00 & 0.93 & 0.08 & 0.90 & 0.95 & 1.000 & 0.362 & 0.124 \\
\hline & 2.00 & 0.92 & 0.09 & 0.89 & 0.95 & & & \\
\hline & 3.00 & 0.96 & 0.09 & 0.93 & 0.98 & & & \\
\hline \multirow[t]{3}{*}{ WHR12 } & 1.00 & 0.88 & 0.09 & 0.85 & 0.91 & 1.000 & 0.563 & 0.252 \\
\hline & 2.00 & 0.87 & 0.11 & 0.84 & 0.90 & & & \\
\hline & 3.00 & 0.91 & 0.10 & 0.88 & 0.94 & & & \\
\hline \multirow[t]{3}{*}{ WHR13 } & 1.00 & 0.92 & 0.11 & 0.88 & 0.95 & 1.000 & 1.000 & 1.000 \\
\hline & 2.00 & 0.92 & 0.10 & 0.89 & 0.95 & & & \\
\hline & 3.00 & 0.94 & 0.11 & 0.91 & 0.97 & & & \\
\hline \multirow[t]{3}{*}{ WHR21 } & 1.00 & 0.92 & 0.08 & 0.90 & 0.95 & 1.000 & 0.101 & 0.028 \\
\hline & 2.00 & 0.91 & 0.08 & 0.88 & 0.94 & & & \\
\hline & 3.00 & 0.96 & 0.09 & 0.93 & 0.99 & & & \\
\hline \multirow[t]{3}{*}{ WHR22 } & 1.00 & 0.87 & 0.08 & 0.85 & 0.90 & 1.000 & 0.440 & 0.257 \\
\hline & 2.00 & 0.86 & 0.10 & 0.83 & 0.90 & & & \\
\hline & 3.00 & 0.90 & 0.10 & 0.87 & 0.93 & & & \\
\hline \multirow[t]{3}{*}{ WHR23 } & 1.00 & 0.90 & 0.10 & 0.88 & 0.93 & 1.000 & 0.684 & 1.000 \\
\hline & 2.00 & 0.91 & 0.10 & 0.88 & 0.95 & & & \\
\hline & 3.00 & 0.93 & 0.11 & 0.90 & 0.97 & & & \\
\hline
\end{tabular}

${ }^{*}$ FDI, notation, $P \leq 0.001, P \leq 0.01$ and $P \leq 0.05$. UAIPMWG: Upper arch inter premolar width groups, WHR: Width/height ratio, 1: Small, 2: Average, 3: Large, Cl: Confidence interval, SD: Standard deviation, FDI: Federation Dentaire Internationale

\begin{tabular}{|c|c|c|c|c|c|c|c|c|}
\hline \multirow[t]{2}{*}{ Variables } & \multirow[t]{2}{*}{ UAIMWG } & \multirow[t]{2}{*}{ Mean } & \multirow[t]{2}{*}{ SD } & \multicolumn{2}{|c|}{$95 \% \mathrm{Cl}$} & \multicolumn{3}{|c|}{$P$} \\
\hline & & & & Lower & Upper & 1 versus 2 & 1 versus 3 & 2 versus 3 \\
\hline \multirow[t]{3}{*}{ WHR11 } & 1.00 & 0.94 & 0.09 & 0.92 & 0.97 & 0.076 & 1.000 & 0.005 \\
\hline & 2.00 & 0.90 & 0.08 & 0.88 & 0.93 & & & \\
\hline & 3.00 & 0.96 & 0.08 & 0.93 & 0.99 & & & \\
\hline \multirow[t]{3}{*}{ WHR12 } & 1.00 & 0.89 & 0.09 & 0.86 & 0.92 & 0.548 & 0.676 & 0.036 \\
\hline & 2.00 & 0.86 & 0.09 & 0.83 & 0.89 & & & \\
\hline & 3.00 & 0.91 & 0.11 & 0.88 & 0.95 & & & \\
\hline \multirow[t]{3}{*}{ WHR13 } & 1.00 & 0.92 & 0.10 & 0.88 & 0.95 & 1.000 & 0.852 & 1.000 \\
\hline & 2.00 & 0.92 & 0.10 & 0.89 & 0.95 & & & \\
\hline & 3.00 & 0.94 & 0.12 & 0.91 & 0.98 & & & \\
\hline \multirow[t]{3}{*}{ WHR21 } & 1.00 & 0.94 & 0.08 & 0.91 & 0.97 & 0.122 & 1.000 & 0.013 \\
\hline & 2.00 & 0.90 & 0.08 & 0.88 & 0.93 & & & \\
\hline & 3.00 & 0.95 & 0.08 & 0.93 & 0.98 & & & \\
\hline \multirow[t]{3}{*}{ WHR22 } & 1.00 & 0.88 & 0.09 & 0.85 & 0.90 & 0.752 & 0.313 & 0.019 \\
\hline & 2.00 & 0.85 & 0.09 & 0.83 & 0.88 & & & \\
\hline & 3.00 & 0.91 & 0.11 & 0.88 & 0.94 & & & \\
\hline \multirow[t]{3}{*}{ WHR23 } & 1.00 & 0.90 & 0.09 & 0.87 & 0.93 & 1.000 & 0.676 & 1.000 \\
\hline & 2.00 & 0.92 & 0.10 & 0.89 & 0.95 & & & \\
\hline & 3.00 & 0.93 & 0.12 & 0.89 & 0.97 & & & \\
\hline
\end{tabular}

${ }^{*}$ FDI, notation, $P \leq 0.001, P \leq 0.01$ and $P \leq 0.05$. UAIMWG: Upper arch inter molar width groups; WHR: Width/height ratio, 1: Small, 2: Average, 3: Large, Cl: Confidence interval, SD: Standard deviation, FDI: Federation Dentaire Internationale

corresponding arch dimension groups values. There were low correlations observed for the maxillary and mandibular arches with $R$ values ranging from 0.17 to 0.42 and 0.26 to 0.29 , respectively.

\section{DISCUSSION}

Current research investigates the crown width/ height ratio in relation to arch perimeter, arch length, and arch width groups (intercanine, interpremolar, and intermolar) for the first time. However, Alam and Iida investigated only mesiodistal tooth size and tooth size ratio in relation to these groups via cone beam computed tomography acquisitions. ${ }^{[31]}$ In orthodontic diagnosis and treatment planning, the evaluation of the tooth size and tooth size discrepancy is an 
Shahid, et al.: Crown width/height ratios and its relation various arch groups

\begin{tabular}{|c|c|c|c|c|c|c|c|c|}
\hline \multirow[t]{2}{*}{ Variables } & \multirow[t]{2}{*}{ LAPG } & \multirow[t]{2}{*}{ Mean } & \multirow[t]{2}{*}{ SD } & \multicolumn{2}{|c|}{$95 \% \mathrm{Cl}$} & \multicolumn{3}{|c|}{$P$} \\
\hline & & & & Lower & Upper & 1 versus 2 & 1 versus 3 & 2 versus 3 \\
\hline \multirow[t]{3}{*}{ WHR31 } & 1.00 & 0.74 & 0.08 & 0.72 & 0.77 & 0.823 & 0.481 & 1.000 \\
\hline & 2.00 & 0.76 & 0.10 & 0.73 & 0.79 & & & \\
\hline & 3.00 & 0.77 & 0.07 & 0.75 & 0.79 & & & \\
\hline \multirow[t]{3}{*}{ WHR32 } & 1.00 & 0.80 & 0.09 & 0.77 & 0.83 & 0.596 & 1.000 & 1.000 \\
\hline & 2.00 & 0.91 & 0.67 & 0.71 & 1.12 & & & \\
\hline & 3.00 & 0.84 & 0.08 & 0.81 & 0.86 & & & \\
\hline \multirow[t]{3}{*}{ WHR33 } & 1.00 & 0.82 & 0.07 & 0.79 & 0.84 & 0.535 & 1.000 & 0.239 \\
\hline & 2.00 & 0.79 & 0.10 & 0.76 & 0.82 & & & \\
\hline & 3.00 & 0.83 & 0.12 & 0.79 & 0.86 & & & \\
\hline \multirow[t]{3}{*}{ WHR41 } & 1.00 & 0.74 & 0.08 & 0.71 & 0.76 & 1.000 & 0.107 & 0.714 \\
\hline & 2.00 & 0.75 & 0.10 & 0.73 & 0.78 & & & \\
\hline & 3.00 & 0.78 & 0.07 & 0.75 & 0.80 & & & \\
\hline \multirow[t]{3}{*}{ WHR42 } & 1.00 & 0.80 & 0.08 & 0.78 & 0.83 & 0.753 & 1.000 & 1.000 \\
\hline & 2.00 & 0.89 & 0.61 & 0.71 & 1.08 & & & \\
\hline & 3.00 & 0.84 & 0.07 & 0.81 & 0.86 & & & \\
\hline \multirow[t]{3}{*}{ WHR43 } & 1.00 & 0.80 & 0.07 & 0.78 & 0.82 & 1.000 & 0.753 & 0.116 \\
\hline & 2.00 & 0.78 & 0.09 & 0.75 & 0.81 & & & \\
\hline & 3.00 & 0.82 & 0.12 & 0.79 & 0.86 & & & \\
\hline
\end{tabular}

${ }^{*} \mathrm{FDI}$, notation, $P \leq 0.001, P \leq 0.01$ and $P \leq 0.05$. LAPG: Lower arch perimeter groups, WHR: Width/height ratio, 1: Small, 2: Average, 3: Large, Cl: Confidence interval, SD: Standard deviation, FDI: Federation Dentaire Internationale

\begin{tabular}{|c|c|c|c|c|c|c|c|c|}
\hline \multirow[t]{2}{*}{ Variables } & \multirow[t]{2}{*}{ LALG } & \multirow[t]{2}{*}{ Mean } & \multirow[t]{2}{*}{ SD } & \multicolumn{2}{|c|}{$95 \% \mathrm{Cl}$} & \multicolumn{3}{|c|}{$P$} \\
\hline & & & & Lower & Upper & 1 versus 2 & 1 versus 3 & 2 versus 3 \\
\hline \multirow[t]{3}{*}{ WHR31 } & 1.00 & 0.75 & 0.09 & 0.72 & 0.78 & 1.000 & 1.000 & 1.000 \\
\hline & 2.00 & 0.76 & 0.09 & 0.73 & 0.78 & & & \\
\hline & 3.00 & 0.77 & 0.08 & 0.74 & 0.79 & & & \\
\hline \multirow[t]{3}{*}{ WHR32 } & 1.00 & 0.91 & 0.67 & 0.70 & 1.12 & 0.638 & 1.000 & 1.000 \\
\hline & 2.00 & 0.80 & 0.08 & 0.78 & 0.83 & & & \\
\hline & 3.00 & 0.84 & 0.10 & 0.81 & 0.87 & & & \\
\hline \multirow[t]{3}{*}{ WHR33 } & 1.00 & 0.79 & 0.10 & 0.76 & 0.82 & 1.000 & 0.219 & 0.479 \\
\hline & 2.00 & 0.80 & 0.09 & 0.77 & 0.83 & & & \\
\hline & 3.00 & 0.83 & 0.11 & 0.80 & 0.87 & & & \\
\hline \multirow[t]{3}{*}{ WHR41 } & 1.00 & 0.75 & 0.08 & 0.72 & 0.77 & 1.000 & 0.643 & 0.777 \\
\hline & 2.00 & 0.75 & 0.09 & 0.72 & 0.78 & & & \\
\hline & 3.00 & 0.77 & 0.08 & 0.75 & 0.79 & & & \\
\hline \multirow[t]{3}{*}{ WHR42 } & 1.00 & 0.90 & 0.61 & 0.71 & 1.09 & 0.545 & 1.000 & 1.000 \\
\hline & 2.00 & 0.80 & 0.07 & 0.78 & 0.82 & & & \\
\hline & 3.00 & 0.83 & 0.08 & 0.80 & 0.86 & & & \\
\hline \multirow[t]{3}{*}{ WHR43 } & 1.00 & 0.80 & 0.10 & 0.77 & 0.83 & 1.000 & 0.981 & 0.311 \\
\hline & 2.00 & 0.79 & 0.08 & 0.76 & 0.81 & & & \\
\hline & 3.00 & 0.82 & 0.11 & 0.78 & 0.85 & & & \\
\hline
\end{tabular}

${ }^{*}$ FDI, notation, $P \leq 0.001, P \leq 0.01$ and $P \leq 0.05$. LALG: Lower arch length groups, WHR: Width/height ratio, 1: Small, 2: Average, 3: Large, Cl: Confidence interval, SD: Standard deviation, FDI: Federation Dentaire Internationale, UALG: Upper arch length groups

essential rung and such investigation was generally determined by conventional plaster study model analysis. ${ }^{[33]}$

The clinical responsibility of the orthodontist is to visualize the macro-, mini-, and micro-esthetics and to design a pleasant smile. ${ }^{[34-36]}$ Current research investigates the crown width/height ratio for the very first time. These investigated norms can be used as a reference in relation to orthodontic and prosthodontic treatment of patients. There was significant difference observed in the worn and nonworn crown width/height ratio. ${ }^{[12]}$ Therefore, in treatment plan, crown width/height ratio must be kept in consideration for the ideal overjet, overbite, and proper interdigitation to be achieved. 
Shahid, et al.: Crown width/height ratios and its relation various arch groups

\begin{tabular}{|c|c|c|c|c|c|c|c|c|}
\hline \multirow[t]{2}{*}{ Variables } & \multirow[t]{2}{*}{ LAICWG } & \multirow[t]{2}{*}{ Mean } & \multirow[t]{2}{*}{ SD } & \multicolumn{2}{|c|}{$95 \% \mathrm{Cl}$} & \multicolumn{3}{|c|}{$P$} \\
\hline & & & & Lower & Upper & 1 versus 2 & 1 versus 3 & 2 versus 3 \\
\hline \multirow[t]{3}{*}{ WHR31 } & 1.00 & 0.76 & 0.09 & 0.74 & 0.79 & 1.000 & 1.000 & 1.000 \\
\hline & 2.00 & 0.75 & 0.09 & 0.72 & 0.78 & & & \\
\hline & 3.00 & 0.76 & 0.08 & 0.74 & 0.78 & & & \\
\hline \multirow[t]{3}{*}{ WHR32 } & 1.00 & 0.82 & 0.10 & 0.79 & 0.85 & 1.000 & 0.594 & 0.463 \\
\hline & 2.00 & 0.81 & 0.09 & 0.78 & 0.84 & & & \\
\hline & 3.00 & 0.93 & 0.67 & 0.72 & 1.14 & & & \\
\hline \multirow[t]{3}{*}{ WHR33 } & 1.00 & 0.80 & 0.10 & 0.77 & 0.83 & 1.000 & 0.570 & 0.495 \\
\hline & 2.00 & 0.80 & 0.07 & 0.78 & 0.82 & & & \\
\hline & 3.00 & 0.83 & 0.11 & 0.79 & 0.86 & & & \\
\hline \multirow[t]{3}{*}{ WHR41 } & 1.00 & 0.76 & 0.08 & 0.73 & 0.78 & 1.000 & 1.000 & 1.000 \\
\hline & 2.00 & 0.75 & 0.09 & 0.72 & 0.78 & & & \\
\hline & 3.00 & 0.76 & 0.08 & 0.73 & 0.78 & & & \\
\hline \multirow[t]{3}{*}{ WHR42 } & 1.00 & 0.81 & 0.09 & 0.78 & 0.83 & 1.000 & 0.437 & 0.467 \\
\hline & 2.00 & 0.81 & 0.09 & 0.78 & 0.83 & & & \\
\hline & 3.00 & 0.92 & 0.61 & 0.73 & 1.11 & & & \\
\hline \multirow[t]{3}{*}{ WHR43 } & 1.00 & 0.79 & 0.09 & 0.76 & 0.82 & 1.000 & 0.570 & 0.423 \\
\hline & 2.00 & 0.79 & 0.07 & 0.77 & 0.81 & & & \\
\hline & 3.00 & 0.82 & 0.12 & 0.78 & 0.86 & & & \\
\hline
\end{tabular}

${ }^{*}$ FDI, notation, $P \leq 0.001, P \leq 0.01$ and $P \leq 0.05$. LAICWG: Lower arch intercanine width groups, WHR: Width/height ratio, 1: Small, 2: Average, 3: Large, Cl: Confidence interval, SD: Standard deviation, FDI: Federation Dentaire Internationale, UAICWG: Upper arch intercanine width groups

\begin{tabular}{|c|c|c|c|c|c|c|c|c|}
\hline \multirow[t]{2}{*}{ Variables } & \multirow[t]{2}{*}{ LAIPMWG } & \multirow[t]{2}{*}{ Mean } & \multirow[t]{2}{*}{ SD } & \multicolumn{2}{|c|}{$95 \% \mathrm{Cl}$} & \multicolumn{3}{|c|}{$P$} \\
\hline & & & & Lower & Upper & 1 versus 2 & 1 versus 3 & 2 versus 3 \\
\hline \multirow[t]{3}{*}{ WHR31 } & 1.00 & 0.74 & 0.07 & 0.72 & 0.76 & 0.136 & 1.000 & 0.874 \\
\hline & 2.00 & 0.78 & 0.10 & 0.75 & 0.81 & & & \\
\hline & 3.00 & 0.76 & 0.08 & 0.73 & 0.78 & & & \\
\hline \multirow[t]{3}{*}{ WHR32 } & 1.00 & 0.81 & 0.09 & 0.78 & 0.83 & 1.000 & 0.541 & 0.858 \\
\hline & 2.00 & 0.83 & 0.10 & 0.80 & 0.86 & & & \\
\hline & 3.00 & 0.92 & 0.67 & 0.71 & 1.13 & & & \\
\hline \multirow[t]{3}{*}{ WHR33 } & 1.00 & 0.79 & 0.08 & 0.76 & 0.82 & 1.000 & 0.211 & 0.898 \\
\hline & 2.00 & 0.81 & 0.09 & 0.78 & 0.84 & & & \\
\hline & 3.00 & 0.83 & 0.12 & 0.79 & 0.87 & & & \\
\hline \multirow[t]{3}{*}{ WHR41 } & 1.00 & 0.74 & 0.07 & 0.72 & 0.76 & 0.250 & 1.000 & 1.000 \\
\hline & 2.00 & 0.77 & 0.10 & 0.74 & 0.80 & & & \\
\hline & 3.00 & 0.75 & 0.08 & 0.73 & 0.78 & & & \\
\hline \multirow[t]{3}{*}{ WHR42 } & 1.00 & 0.80 & 0.08 & 0.77 & 0.82 & 1.000 & 0.417 & 0.720 \\
\hline & 2.00 & 0.82 & 0.09 & 0.79 & 0.85 & & & \\
\hline & 3.00 & 0.91 & 0.61 & 0.72 & 1.10 & & & \\
\hline \multirow[t]{3}{*}{ WHR43 } & 1.00 & 0.78 & 0.08 & 0.75 & 0.80 & 0.814 & 0.061 & 0.488 \\
\hline & 2.00 & 0.80 & 0.09 & 0.77 & 0.83 & & & \\
\hline & 3.00 & 0.83 & 0.12 & 0.79 & 0.86 & & & \\
\hline
\end{tabular}

*FDI, notation, $(P \leq 0.001),(P \leq 0.01)$ and $(P \leq 0.05)$. LAIPMWG: Lower arch inter premolar width groups, WHR: Width/height ratio, 1: Small, 2: Average, 3: Large, $\mathrm{Cl}$ : Confidence interval, SD: Standard deviation, FDI: Federation Dentaire Internationale

Through our study, we found out that there is significant sexual difference in the Pakistani population in the mean crown width/height ratios of maxillary and mandibular anterior six teeth $(P \leq 0.05)$. However, there were no significant differences observed in relation to the arch length, arch perimeter, and arch width groups $(P \geq 0.05)$. As study on Bangladeshi population reported no significant difference in the mean crown width/ height ratios of maxillary anterior teeth between the various facial groups $(P>0.05) .{ }^{[24]}$

Our research investigated the maxillary and mandibular anterior teeth width/height ratio with ideal overjet, overbite, and proper interdigitation in relation to arch perimeter, arch length, and arch width groups (intercanine, interpremolar, and intermolar). 
Shahid, et al.: Crown width/height ratios and its relation various arch groups

\begin{tabular}{|c|c|c|c|c|c|c|c|c|}
\hline \multirow[t]{2}{*}{ Variables } & \multirow[t]{2}{*}{ LAIMWG } & \multirow[t]{2}{*}{ Mean } & \multirow[t]{2}{*}{ SD } & \multicolumn{2}{|c|}{$95 \% \mathrm{Cl}$} & \multicolumn{3}{|c|}{$P$} \\
\hline & & & & Lower & Upper & 1 versus 2 & 1 versus 3 & 2 versus 3 \\
\hline \multirow[t]{3}{*}{ WHR31 } & 1.00 & 0.75 & 0.10 & 0.72 & 0.78 & 1.000 & 1.000 & 1.000 \\
\hline & 2.00 & 0.76 & 0.07 & 0.74 & 0.78 & & & \\
\hline & 3.00 & 0.77 & 0.08 & 0.74 & 0.79 & & & \\
\hline \multirow[t]{3}{*}{ WHR32 } & 1.00 & 0.81 & 0.11 & 0.78 & 0.84 & 1.000 & 0.401 & 0.505 \\
\hline & 2.00 & 0.82 & 0.08 & 0.79 & 0.84 & & & \\
\hline & 3.00 & 0.94 & 0.69 & 0.72 & 1.16 & & & \\
\hline \multirow[t]{3}{*}{ WHR33 } & 1.00 & 0.79 & 0.10 & 0.77 & 0.82 & 1.000 & 0.087 & 0.176 \\
\hline & 2.00 & 0.80 & 0.09 & 0.77 & 0.83 & & & \\
\hline & 3.00 & 0.84 & 0.11 & 0.80 & 0.88 & & & \\
\hline \multirow[t]{3}{*}{ WHR41 } & 1.00 & 0.75 & 0.09 & 0.72 & 0.77 & 1.000 & 0.761 & 1.000 \\
\hline & 2.00 & 0.75 & 0.08 & 0.73 & 0.78 & & & \\
\hline & 3.00 & 0.77 & 0.08 & 0.74 & 0.79 & & & \\
\hline \multirow[t]{3}{*}{ WHR42 } & 1.00 & 0.80 & 0.09 & 0.77 & 0.83 & 1.000 & 0.312 & 0.466 \\
\hline & 2.00 & 0.81 & 0.08 & 0.79 & 0.84 & & & \\
\hline & 3.00 & 0.93 & 0.63 & 0.72 & 1.13 & & & \\
\hline \multirow[t]{3}{*}{ WHR43 } & 1.00 & 0.79 & 0.10 & 0.76 & 0.82 & 1.000 & 0.108 & 0.115 \\
\hline & 2.00 & 0.79 & 0.07 & 0.76 & 0.81 & & & \\
\hline & 3.00 & 0.83 & 0.11 & 0.79 & 0.87 & & & \\
\hline
\end{tabular}

${ }^{*} F D I$, notation, $(P \leq 0.001),(P \leq 0.01)$ and $(P \leq 0.05)$. LAIMWG: Lower arch inter molar width groups, WHR: Width/height ratio, 1: Small, 2: Average, 3: Large, Cl: Confidence interval, SD: Standard deviation, FDI: Federation Dentaire Internationale

The research results showed no significant difference in relation to these groups of ideal occlusion [Tables 3-11]. Other studies investigated the ideal occlusion in relation to maxillary and mandibular tooth size ratios and found significant differences in relation to these groups. ${ }^{[31]}$ But current research found no significant difference for crown width/height ratio to various arch groups and low correlations [Table 12].

For the Pakistani population the tooth were investigated in mesiodistal, buccolingual, and diagonal dimension. ${ }^{[37]}$

The upshot of this study would be advantageous for both manufacturer and clinician. The current data can be used as reference, in order to create a natural pleasant esthetic smile and look. Especially during planning orthodontic, implant therapies, periodontal surgeries, and dental prosthesis procedures involving maxillary and mandibular esthetic zone. Currently, there are many manufacturers' products of artificial teeth in various dimensions. Not all products are suitable for every person as there are variations in natural tooth size and shape from one person to another. By using the crown width/height ratio values obtained in this study, the proposed width/height, and there ratio can be calculated for each ethnic group and thus may accurately determine the ideal tooth shape and size in the esthetic zone. Therefore, such investigations are needed to be carried out in other population. Furthermore the norms will be of great
Table 12: Correlation coefficients $(R)$ and coefficients of determination $\left(R^{2}\right)$ between maxillary crown WHR and its relation to various arch dimensions

\begin{tabular}{lccc}
\hline Variables & $\boldsymbol{R}$ and $\boldsymbol{R}^{\mathbf{2}}$ & Max 3-3 CWHR & Mand 3-3 CWHR \\
\hline APgp & $R$ & 0.20 & 0.27 \\
\multirow{3}{*}{ ALgp } & $R^{2}$ & 0.04 & 0.07 \\
\multirow{4}{*}{ ICWgp } & $R$ & 0.17 & 0.27 \\
& $R^{2}$ & 0.03 & 0.07 \\
IPMWgp & $R$ & 0.42 & 0.24 \\
& $R$ & 0.18 & 0.06 \\
IMWgp & $R^{2}$ & 0.24 & 0.29 \\
& $R$ & 0.06 & 0.08 \\
& $R^{2}$ & 0.17 & 0.26 \\
& $R$ & 0.03 & 0.07
\end{tabular}

APgp: Arch perimeter groups, ALgp: Arch length groups, ICWgp: Intercanine width groups, IPMWgp: Inter premolar width groups, IMWgp: Inter molar width groups, Max 3-3 CWHR: Maxillary canine to canine crown WHR, Mand 3-3 CWHR: Mandibular canine to canine crown WHR correlation coefficients, $R^{2}$ : Coefficients of determination, WHR: Width/height ratio

value in forensic dentistry, and dental anthropology. Human teeth and arch size have lots of variations in size in relation to culture, race, and sex..$^{[11,37,38]}$

\section{CONCLUSION}

- Significant sexual dimorphisms were observed in the crown width/height ratios of maxillary and mandibular anterior teeth in few variables

- There were significant differences observed for crown width/height ratios of maxillary arch Intercanine and Inter first molar arch width groups (small vs. large and average vs. large) 
- There were no significant differences observed for crown width/height ratios of maxillary and mandibular anterior tooth in relation to:

- Maxillary and mandibular arch perimeter groups

- Maxillary and mandibular arch length groups

- Mandibular arch width inter first premolar width groups

- Mandibular arch width groups in:

- Intercanine width groups

- Inter first premolar width groups

- Inter first molar width groups

\section{Acknowledgment}

The authors would like to acknowledge the support from the USM 304/PPSG/ 61313104 short-term grant.

\section{Financial support and sponsorship}

Universiti Sains Malaysia 304/PPSG/61313104 short-term grant.

\section{Conflicts of interest}

There are no conflicts of interest.

\section{REFERENCES}

1. Yonezu T, Warren JJ, Bishara SE, Steinbock KL. Comparison of tooth size and dental arch widths in contemporary Japanese and American preschool children. World J Orthod 2001;2:356-60.

2. Lavelle CL. Maxillary and mandibular tooth size in different racial groups and in different occlusal categories. Am J Orthod 1972;61:29-37.

3. Smith SS, Buschang PH, Watanabe E. Interarch tooth size relationships of 3 populations: "Does Bolton's analysis apply?" Am J Orthod Dentofacial Orthop 2000;117:169-74.

4. Ta TA, Ling JY, Hägg U. Tooth-size discrepancies among different occlusion groups of southern Chinese children. Am J Orthod Dentofacial Orthop 2001;120:556-8.

5. Araujo E, Souki M. Bolton anterior tooth size discrepancies among different malocclusion groups. Angle Orthod 2003;73:307-13.

6. Proffit WR, Fields HW Jr, Sarver DM. Contemporary Orthodontics. Philadelphia, USA: Elsevier Health Sciences; 2006.

7. Lee GT. Ethnic variations in teeth morphology. Proc Br Paedod Soc 1977;7:23-7.

8. Yaacob H, Nambiar P, Naidu MD. Racial characteristics of human teeth with special emphasis on the Mongoloid dentition. Malays J Pathol 1996;18:1-7.

9. Younes SA, al-Shammery AR, el-AngbawiMF. Anatomic measurements of teeth in two different racial groups from the Middle East. Egypt Dent J 1988;34:371-8, 380-4, 386-91.

10. Ribeiro DC, Brook AH, Hughes TE, Sampson WJ, Townsend GC. Intrauterine hormone effects on tooth dimensions. J Dent Res 2013;92:425-31.

11. Townsend G, Bockmann M, Hughes T, Brook A. Genetic, environmental and epigenetic influences on variation in human tooth number, size and shape. Odontology 2012;100:1-9.

12. Tsukiyama T, Marcushamer E, Griffin TJ, Arguello E, Magne P, Gallucci GO. Comparison of the anatomic crown width/length ratios of unworn and worn maxillary teeth in Asian and white subjects. J Prosthet Dent 2012;107:11-6.

13. Bailit HL. Dental variation among populations. An anthropologic view. Dent Clin North Am 1975;19:125-39.

14. Lombardi RE. The principles of visual perception and their clinical application to denture esthetics. J Prosthet Dent 1973;29:358-82.

15. Levin EI. Dental esthetics and the golden proportion. J Prosthet Dent 1978;40:244-52.
16. Qualtrough AJ, Burke FJ. A look at dental esthetics. Quintessence Int 1994;25:7-14.

17. Rosenstiel SF, Ward DH, Rashid RG. Dentists' preferences of anterior tooth proportion: A web-based study. Int J Prosthodont 2000;9:123-36.

18. Ward DH. Proportional smile design using the recurring esthetic dental (red) proportion. Dent Clin North Am 2001;45:143-54.

19. Gurel G. The Science and Art of Porcelain Laminate Veneers. London: Quintessence; 2003. p. 83-6.

20. Scandrett FR, Kerber PE, Umrigar ZR. A clinical evaluation of techniques to determine the combined width of the maxillary anterior teeth and the maxillary central incisor. J Prosthet Dent 1982;48:15-22.

21. Cesario VA Jr, Latta GH Jr. Relationship between the mesiodistal width of the maxillary central incisor and interpupillary distance. J Prosthet Dent 1984;52:641-3.

22. Latta GH Jr, Weaver JR, Conkin JE. The relationship between the width of the mouth, interalar width, bizygomatic width, and interpupillary distance in edentulous patients. J Prosthet Dent 1991;65:250-4.

23. Abdullah MA. Inner canthal distance and geometric progression as a predictor of maxillary central incisor width. J Prosthet Dent 2002;88:16-20.

24. Nafij BJ, Nita V, Pokpong A, Suchaya P, Jira C, Alam MK. Comparison of crown width/length ratio of six maxillary anterior teeth between different facial groups in Bangladeshi population. Int Med J 2014;21:49-54.

25. Vandenbroucke JP, von Elm E, Altman DG, Gøtzsche PC, Mulrow CD, Pocock SJ, et al. Strengthening the reporting of observational studies in epidemiology (STROBE): Explanation and elaboration. Ann Intern Med 2007;147:W163-94.

26. Prabhu S, Acharya AB. Odontometric sex assessment in Indians. Forensic Sci Int 2009;192:129.e1-5.

27. Dupont WD, Plummer WD Jr. Power and sample size calculations for studies involving linear regression. Control Clin Trials 1998;19:589-601.

28. Williams AC, Stephens CD. A modification to the incisor classification of malocclusion. Br J Orthod 1992;19:127-30.

29. Shahid F, Alam MK, Khamis MF, Muraoka R, Nakano K, Okafuji N. Validity and reliability of digital model measurements: A digital stereomicroscopic study. J Hard Tissue Biol 2014;23:439-44.

30. Volchansky A, Cleaton-Jones P. Clinical crown height (length) - A review of published measurements. J Clin Periodontol 2001;28:1085-90.

31. Alam MK, Shahid F, Purmal K, Ahmad B, Khamis MF. Bolton tooth size ratio and its relation with arch widths, arch length and arch perimeter: A cone beam computed tomography (CBCT) study. Acta Odontol Scand 2014;72:1047-53.

32. Houston WJ. The analysis of errors in orthodontic measurements. Am J Orthod 1983;83:382-90.

33. Alam MK, Iida J. Overjet, overbite and dental midline shift as predictors of tooth size discrepancy in a Bangladeshi population and a graphical overview of global tooth size ratios. Acta Odontol Scand 2013;71:1520-31

34. Djeu G, Hayes C, Zawaideh S. Correlation between mandibular central incisor proclination and gingival recession during fixed appliance therapy. Angle Orthod 2002;72:238-45.

35. Bergman RT, Waschak J, Borzabadi-Farahani A, Murphy NC. Longitudinal study of cephalometric soft tissue profile traits between the ages of 6 and 18 years. Angle Orthod 2014;84:48-55.

36. Sterrett JD, Oliver T, Robinson F, Fortson W, Knaak B, Russell CM. Width/length ratios of normal clinical crowns of the maxillary anterior dentition in man. J Clin Periodontol 1999;26:153-7.

37. Shahid F, Alam MK, Khamis MF, Honda Y, Sugita Y, Maeda H. Geomorphometrics of tooth size and arch dimension analysis by conventional digital caliper and digital stereomicroscope to establish standard norms for the Pakistani population. J Hard Tissue Biol 2015;24:155-68

38. Mihailidis S, Scriven G, Khamis M, Townsend G. Prevalence and patterning of maxillary premolar accessory ridges (MxPARs) in several human populations. Am J Phys Anthropol 2013;152:19-30.

\begin{tabular}{|l|l|}
\hline \multicolumn{2}{|c|}{ Access this article online } \\
\hline Quick Response Code: \\
\hline
\end{tabular}

